\title{
Insomnia and cardiorespiratory fitness in a middle-aged population: the SCAPIS pilot study
}

\author{
Ding Zou ${ }^{1}\left(\mathbb{D} \cdot\right.$ Heini Wennman $^{2} \cdot$ Örjan Ekblom $^{3} \cdot$ Ludger Grote $^{1} \cdot$ Daniel Arvidsson $^{4} \cdot$ Anders Blomberg $^{5}$. \\ Kjell Torén ${ }^{6} \cdot$ Göran Bergström ${ }^{7,8} \cdot$ Mats Börjesson $^{4,8} \cdot$ Jan Hedner $^{1}$
}

Received: 29 June 2018 / Revised: 6 November 2018 / Accepted: 27 November 2018 / Published online: 13 December 2018

(C) The Author(s) 2018

\begin{abstract}
Background The relationship between insomnia and cardiorespiratory fitness (CRF), a well-established risk factor for cardiovascular disease, has not been extensively studied. We aimed to assess the independent association between insomnia and CRF in a population-based cohort of subjects aged 50 to 64 years.

Methods Subjects participating in the Swedish CArdioPulmonary bioImaging Study (SCAPIS) pilot cohort ( $n=603$, men $47.9 \%$ ) underwent a submaximal cycle ergometer test for estimation of maximal oxygen consumption $\left(\mathrm{VO}_{2} \mathrm{max}\right)$. Data on physical activity and sedentary time were collected via waist-worn accelerometers. An insomnia severity index score $\geq 10$ was used to define insomnia.

Results Insomnia was identified in $31.8 \%$ of the population. The $\mathrm{VO}_{2}$ max was significantly lower in insomnia subjects compared with the non-insomnia group (31.2 \pm 6.3 vs. $\left.32.4 \pm 6.5 \mathrm{ml}^{*} \mathrm{~kg}^{-1} * \mathrm{~min}^{-1}, p=0.028\right)$. There was no difference in objectively assessed physical activity or time spent sedentary between the groups. In a multivariate generalized linear model adjusting for confounders, an independent association between insomnia status and lower $\mathrm{VO}_{2}$ max was found in men, but not in women $(\beta=$ -1.15 [95\% CI $-2.23-0.06$ ] and -0.09 [-1.09-0.92], $p=0.038$ and 0.866 , respectively).

Conclusions We found a modest, but significant, association between insomnia and lower CRF in middle-aged men, but not in women. Our results suggest that insomnia may link to cardiovascular disease via reduced CRF. Insomnia may require a specific focus in the context of health campaigns addressing CRF.
\end{abstract}

Keywords Cardiovascular disease · Gender · Insomnia · Maximal oxygen consumption · Physical activity · Population-based cohort

Ding Zou and Heini Wennman contributed equally to this work.

Electronic supplementary material The online version of this article (https://doi.org/10.1007/s11325-018-1765-9) contains supplementary material, which is available to authorized users.

Ding Zou

zou.ding@lungall.gu.se

1 Center for Sleep and Vigilance Disorders, Department of Internal Medicine and Clinical Nutrition, Sahlgrenska Academy, University of Gothenburg, Medicinaregatan 8b, Box 421, SE-40530 Gothenburg, Sweden

2 Department of Public Health Solutions, National Institute for Health and Welfare, Helsinki, Finland

3 Åstrand Laboratory of Work Physiology, The Swedish School of Sport and Health Sciences, Stockholm, Sweden
4 Center for Health and Performance, Department of Food and Nutrition, and Sport Science, Institute of Neuroscience and Physiology, University of Gothenburg, Gothenburg, Sweden

5 Department of Public Health and Clinical Medicine, Division of Medicine/Respiratory Medicine, Umeå University, Umeå, Sweden

6 Section of Occupational and Environmental Medicine, Sahlgrenska Academy, University of Gothenburg, Gothenburg, Sweden

7 Department of Molecular and Clinical Medicine, Institute of Medicine, Sahlgrenska Academy, University of Gothenburg, Gothenburg, Sweden

8 Sahlgrenska University Hospital, Gothenburg, Sweden 


\section{Background}

Insomnia is defined as problems with sleep initiation, consolidation, duration, or quality, despite an opportunity to sleep enough coupled with daytime impairment. Insomnia and insomnia-related symptoms are highly prevalent in adult populations, ranging from 5 to $30 \%$, being more common among women than men and increasing with age [1]. During the past decades, the prevalence of insomnia symptoms has grown both in the USA and Europe [2-4] and mounting evidence shows that insomnia is highly comorbid with psychiatric disorders, e.g., depression [1]. Emerging data also suggest that insomnia constitutes a risk factor for cardiometabolic diseases $[5,6]$. Several mechanisms have been proposed to link insomnia and cardiometabolic disease including endocrine dysregulation, inflammation, autonomic imbalance, and endothelial dysfunction [6,7]. It is also suggested that subjects with insomnia symptoms engage in more adverse health behaviors such as smoking and alcohol consumption and are less physically active $[2,8]$.

Cardiorespiratory fitness (CRF) describes the capacity of the circulatory system to provide working muscles with oxygen during heavy working loads and is often expressed in terms of measured or estimated maximal oxygen consumption $\left(\mathrm{VO}_{2} \mathrm{max}\right)$ level [9]. Higher levels of CRF have been shown to associate with numerous health benefits including reduced burden of anxiety and depression [10]. Conversely, low levels of CRF predict increased cardiometabolic disease risk and allcause mortality [11].

Studies addressing the association between insomnia and CRF are sparse. In a large community-based sample, there was a moderate inverse association between self-reported insomnia symptoms and CRF, assessed by peak oxygen uptake [12]. The total score on the Insomnia Severity Index (ISI) questionnaire was inversely associated with self-perceived fitness in a cohort of students [13]. Therefore, in the current study, we aimed to investigate the association between insomnia classified by ISI and CRF assessed by a submaximal exercise test in a population-based sample of middle-aged, urban Swedish men and women. We hypothesized that insomnia is associated with decreased $\mathrm{VO}_{2}$ max, independently of objectively measured physical activity level.

\section{Material and methods}

\section{Overview of the study cohort}

The Swedish CArdioPulmonary bioImage Study (SCAPIS) is an ongoing national multicenter study with the focus on identification and investigation of cardiometabolic disease and related mechanisms in a cohort of 30,000 men and women, aged 50 to 64 years [14]. In 2012, a comprehensive pilot trial was conducted at the Sahlgrenska University Hospital in Gothenburg, Sweden, to examine the feasibility and financial and ethical consequences of SCAPIS. In detail, 2243 subjects were randomly selected from the Swedish population register stratified for low and high socioeconomic status (SES) area and 1111 agreed to participate. Participants underwent a 2-day protocol including imaging and functional tests of the heart, lungs, and metabolism as well as extensive health questionnaires. In addition, participants were asked to perform a submaximal fitness test and to wear an accelerometer during 7 days to objectively record daily physical activity. All participants gave their written informed consent and SCAPIS has been approved as a multicenter study by the ethics committee at Umeå University (Dnr 2010-228-31M). The protocol for the current study was approved by the regional Ethics Committee of Gothenburg (Dnr 638-16).

\section{Insomnia and sleep duration}

The Insomnia Severity Index questionnaire was used to classify insomnia [15]. The ISI consists of 7 questions assessing the nature, severity, and impact of insomnia (total score ranges from 0 to 28). A cutoff of ISI score $\geq 10$ was applied for insomnia diagnosis [16]. Subjective sleep duration was assessed by the question: "How many hours do you usually sleep during 24-hours" with response alternatives: "4 hours or less; 5 hours; 6 hours; 7 hours; 8 hours; 9 hours; 10 hours or more," further divided into a categorical variable "subjective sleep duration $<6$ hours" yes or no.

\section{Cardiorespiratory fitness and physical activity}

Participants were invited to participate in a submaximal cycle ergometer exercise test for the assessment of $\mathrm{VO}_{2}$ max. The test followed the Ekblom-Bak cycle ergometer protocol for adults [17] and included 4 min cycling (Monark ergometer 828E, Monark Exercise AB, Vansbro, Sweden) at two different workloads, respectively. The first workload was standard and low work rate $(\sim 30 \mathrm{~W})$ and the second a higher, individually chosen work rate aiming for a perceived exertion of approximately 14 on the Borg scale [18]. The mean heart rate (Polar Electro Oy, Kempele, Finland) during the last minute at the low and high workload was recorded respectively. $\mathrm{VO}_{2}$ max was estimated based on the difference in workload and heart rate between the two workloads using validated gender-specific equations [19]. Subjects with diagnosed heart condition, taking beta-adrenergic blockers, having pain in hips, back, or knees, with obesity or perceived inability to perform the test were excluded from the test.

Objective measurement of physical activity in the SCAPIS pilot trial has been described in detail previously [20]. Briefly, participants were instructed to wear the triaxial accelerometers (ActiGraph GT3X and GT3X+, Actigraph, LCC, Pensacola, 
FL, USA) on the right hip for seven consecutive days during waking hours but not when in water-based activities. Sampling frequency was set at $30 \mathrm{~Hz}$ and data were downloaded using the ActiLife software (v. 6.10.1, Actigrah LCC, Pensacola, FL, USA) as 60-s epochs with low frequency extension filtering. Participants with at least $600 \mathrm{~min}$ of accelerometer wear time for at least 4 days were accepted to be included in analyses. The vector magnitude counts per minute (cpm) were used to classify time spent sedentary (SED: 0$199 \mathrm{cpm}$ ) and moderate to vigorous intensity physical activity (MVPA: $\geq 2690 \mathrm{cpm}$ ) [21, 22]. Data for SED and MVPA are shown as mean daily percentage over the measurement period.

\section{Information on lifestyle, living conditions, and comorbidities}

Weight $(\mathrm{kg})$, height $(\mathrm{m})$, and waist circumference $(\mathrm{cm})$ were measured prior to the fitness test. Body mass index (BMI) was calculated as weight divided by square height $\left(\mathrm{kg} / \mathrm{m}^{2}\right)$. Selfreported smoking habit was classified as never smoker, occasional smoker, former smoker, and current active smoker. Alcohol consumption was assessed using the Alcohol Use Disorders Identification Test consumption (AUDIT-c) questionnaire where a total score of 5 or higher in men and 4 or higher in women was classified as risk drinking [23].

University education (yes; no) was used to describe the subjects' education level. Participants were asked if they currently are engaged in an income-related job or not.

Self-reported chronic disease was identified for subjects who reported one or more of the following: diagnosed diabetes, cancer, or COPD, previous bypass surgery or hospitalization because of myocardial infarction/stroke. The question "Have you ever had clinically diagnosed sleep apnea (yes/no)?" was used to identify subjects with comorbid sleep apnea. Information about depression symptoms was based on the question: "During the past 12 months, have you experienced a period of two or more than two weeks when you have felt sad, downhearted or depressed? (yes/no)."

\section{Statistical analysis}

Statistical analyses were performed using SPSS 25.0 (IBM, Armonk, NY, USA). Data are shown as mean (SD) or median [interquartile range IQR]. Distribution of the variables was analyzed using Kolmogorov-Smirnov test. Chi-square tests were used to compare categorical variables. Normally distributed variables were compared using independent sample $t$ test and Mann-Whitney test was applied for non-normally distributed data comparison. Effect size of the independent $t$ test was determined by Cohen's d. The association between insomnia and CRF was analyzed using generalized linear models, adjusting for age, gender, BMI, waist circumference, SES, university education, income-related job, smoking, risky drinking, chronic disease, sleep apnea, depression symptoms, sleep duration < $6 \mathrm{~h}$, percentage of MVPA, and percentage of SED. Subgroup analyses were performed stratified by gender. Sensitivity analyses were performed by excluding participants who reported symptoms of depression or were identified with chronic disease. A $p$ value $<0.05$ was considered statistically significant.

\section{Results}

\section{Description of the study population}

Out of 1111 subjects in the SCAPIS pilot study, 795 participants (71.6\% of full sample) performed a submaximal cycle ergometer exercise test. Of these subjects, 82 subjects had no/ incomplete ISI score, 74 did not complete the accelerometer test, and 15 subjects lacked both assessments. Six hundred twenty-four had complete information on both the ISI questionnaire and the objectively measured physical activity. Subjects with missing information on income-related job, risky drinking, self-reported chronic disease/sleep apnea/depression symptoms, or subjective sleep duration were further excluded from the analyses $(n=21)$. The final cohort included 603 subjects (289 men; mean age $57 \pm 4$ years; BMI $26.8 \pm$ $\left.4.0 \mathrm{~kg} / \mathrm{m}^{2}\right)$.

\section{Non-response analysis}

Compared to the final cohort the excluded subjects were slightly older, more overweight, more sedentary and more often current smokers. As expected, self-reported chronic disease, depression symptoms, insomnia, low socioeconomic status, and no university degree as well as no income-related job were more prevalent among these subjects (supplementary table 1). There were no significant differences in $\mathrm{VO}_{2} \mathrm{max}$ or MVPA between the two groups.

\section{Insomnia vs. non-insomnia}

The prevalence of insomnia (ISI $\geq 10$ ) was $31.8 \%$ in this middle-aged population. The median ISI score in men and women was 6 [IQR 2 to 10] and 7 [4 to 12], respectively (Mann-Whitney test, $p<0.001$ ). The proportion of ISI $<10$ in women and men was $64.3 \%$ and $72.3 \%$, respectively. As expected, insomnia was more prevalent among women than men $(35.7 \%$ vs. $27.7 \%, p=0.035)$. Compared to the non-insomnia group, there was a higher proportion of low socioeconomic status, no income-related job and depression symptoms in the insomnia group (all $p<0.01)$. 
There were no significant differences in objectively measured physical activity (MVPA) or SED between the groups. However, $\mathrm{VO}_{2} \max$ was lower in insomnia compared to non-insomnia subjects $(31.2 \pm 6.3$ vs. $32.4 \pm$ $6.5 \mathrm{ml} * \mathrm{~kg}^{-1} * \mathrm{~min}^{-1}, p=0.028$, Cohen's $d=0.49$, Table 1). Insomnia was consistently associated with lower $\mathrm{VO}_{2} \max$ across the tertiles of MVPA, and $\mathrm{VO}_{2} \max$ showed a significant difference in subjects with the highest MVPA level (Fig. 1).

\section{The association between insomnia and $\mathrm{VO}_{2}$ max}

In a multivariate generalized linear model, insomnia status was not independently associated with $\mathrm{VO}_{2} \max (\beta=-0.56$, 95\% CI $-1.31-0.20, p=0.148$ ) controlling for age, gender, BMI, waist circumference, SES, university education, income-related job, smoking, risky alcohol consumption, subjective short sleep duration $<6 \mathrm{~h}$, depression symptoms, selfreported chronic disease, sleep apnea, percentage of MVPA, and percentage of SED (Table 2). However, in a genderstratified multivariate analysis, insomnia was significantly associated with lower $\mathrm{VO}_{2} \max$ in men $(\beta=-1.15,95 \% \mathrm{CI}-$ $2.23-0.06, p=0.038)$, but not in women $(\beta=-0.09,95 \%$ CI $-1.09-0.92, p=0.866$ ) (Table 3).

\section{Sensitivity analyses}

In a repeated sensitivity analysis excluding 66 subjects with self-reported chronic disease, the result between insomnia and $\mathrm{VO}_{2}$ max remained unchanged (supplementary tables 2 and 3 ). Similarly, when excluding subjects with self-reported symptoms of depression $(n=145)$, the association between insomnia and lower $\mathrm{VO}_{2}$ max in men was preserved (supplementary tables 4 and 5).

\section{Discussion}

In this middle-aged gender-balanced cohort, insomnia detected by ISI score $\geq 10$ was associated with lower CRF, particularly in men. This association was independent of SES, body composition, comorbidity, and lifestyle including MVPA. To the best of our knowledge, this is the first study using a valid insomnia diagnosis instrument rather than insomnia symptoms to address the link between CRF and insomnia. It is argued that in relation to, and possibly in addition to, unhealthy lifestyle (e.g., physical inactivity) low CRF may constitute an important link to increased cardiometabolic risk in insomnia.

Table 1 Characteristics of the studied cohort by insomnia status $(n=603)$

\begin{tabular}{|c|c|c|c|c|}
\hline & Total & $\begin{array}{l}\text { Insomnia severity } \\
\text { index }<10(n=411)\end{array}$ & $\begin{array}{l}\text { Insomnia severity } \\
\text { index } \geq 10(n=192)\end{array}$ & $\begin{array}{l}p \text { value for } \\
\text { comparison }\end{array}$ \\
\hline $\operatorname{Men}(\%)$ & 47.9 & 50.9 & 41.7 & 0.035 \\
\hline Age (years) & $57.2(4.4)$ & $57.4(4.4)$ & $56.9(4.1)$ & 0.148 \\
\hline Body mass index $\left(\mathrm{kg} / \mathrm{m}^{2}\right)$ & $26.8(4.0)$ & $26.6(3.9)$ & $27.2(4.3)$ & 0.137 \\
\hline Waist circumference $(\mathrm{cm})$ & $93.7(11.4)$ & $93.7(11.5)$ & $93.7(11.3)$ & 0.989 \\
\hline Low socioeconomic status (\%) & 39.8 & 35.8 & 48.4 & 0.003 \\
\hline University degree (\%) & 42.6 & 45.3 & 37.0 & 0.056 \\
\hline No income-related job (\%) & 17.4 & 13.6 & 25.5 & $<0.001$ \\
\hline \multicolumn{5}{|l|}{ Smoking (\%) } \\
\hline Never & 47.1 & 48.9 & 43.2 & 0.339 \\
\hline Occasional & 2.8 & 3.2 & 2.1 & \\
\hline Former & 40.0 & 38.9 & 42.2 & \\
\hline Current & 10.1 & 9.0 & 12.5 & \\
\hline Risky alcohol consumption (\%) & 28.9 & 27.5 & 31.8 & 0.280 \\
\hline Subjective sleep duration $<6 \mathrm{~h}(\%)$ & 9.0 & 3.2 & 21.4 & $<0.001$ \\
\hline Sleep apnea $(\%)$ & 4.3 & 3.9 & 5.2 & 0.459 \\
\hline Chronic disease $(\%)$ & 10.9 & 9.5 & 14.1 & 0.094 \\
\hline Depression symptoms (\%) & 24.0 & 16.1 & 41.1 & $<0.001$ \\
\hline Percentage MVPA & $5.7[3.7]$ & $5.5[3.6]$ & $5.9[3.6]$ & 0.358 \\
\hline Percentage SED & $52.6(9.8)$ & $52.4(9.9)$ & $52.8(9.6)$ & 0.616 \\
\hline $\mathrm{VO}_{2} \max \left(\mathrm{ml} * \min ^{-1} * \mathrm{~kg}^{-1}\right)$ & $32.0(6.5)$ & $32.4(6.5)$ & $31.2(6.3)$ & 0.028 \\
\hline
\end{tabular}

Data show as mean (SD) or median [inter quartile range]. Chronic disease includes stroke, coronary artery disease, diabetes, cancer, and COPD

Significant $p$ value presented in italics

MVPA moderate to vigorous intensity physical activity, SED time spent sedentary 
Fig. $1 \mathrm{VO}_{2} \max$ by insomnia and non-insomnia groups across MVPA tertiles

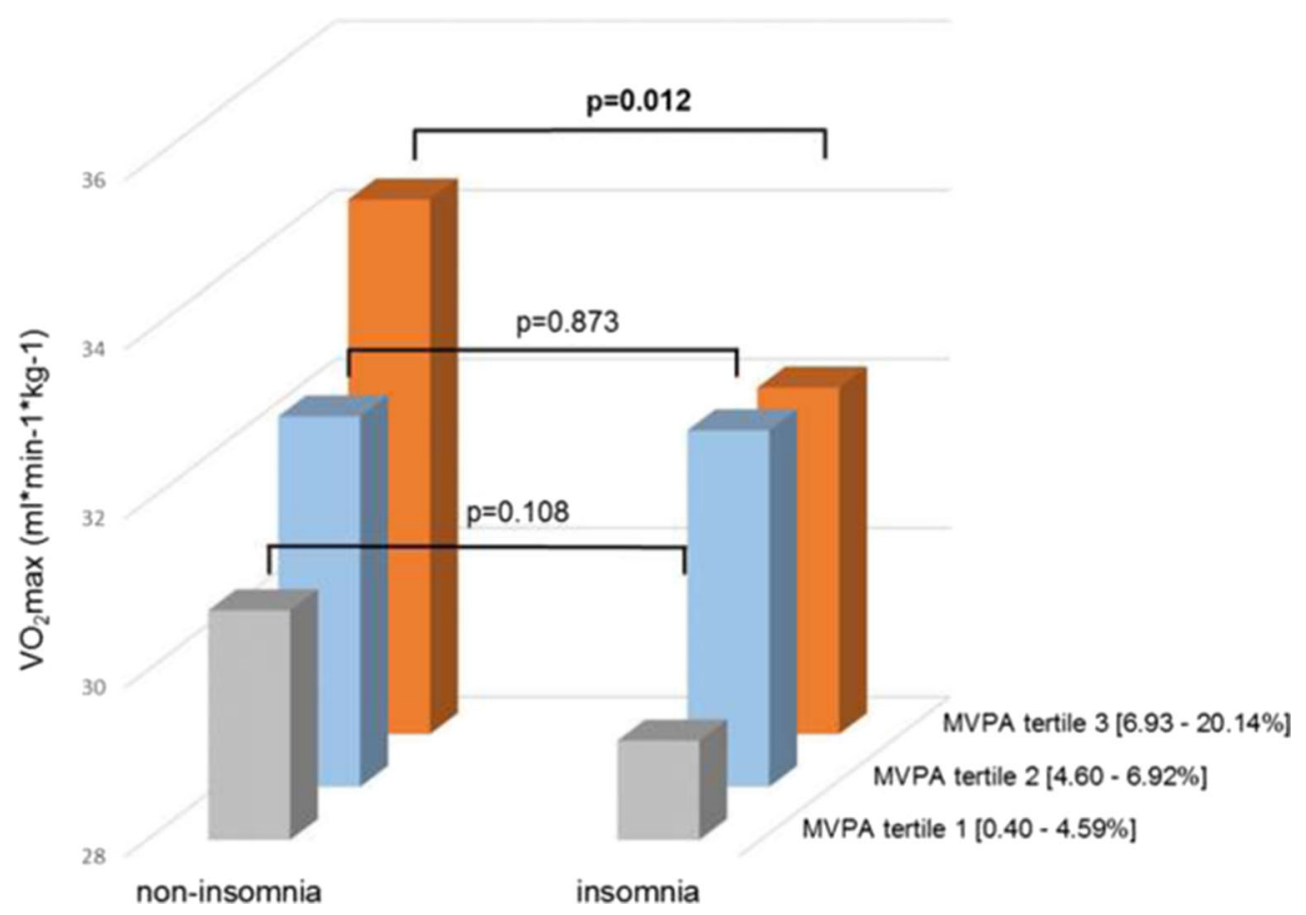

Both physical activity and CRF provide strong prognostic ability for future cardiovascular disease. CRF is moderately related to the intense aspect of habitual physical activity and has been observed to be a stronger predictor of cardiovascular events and mortality than physical activity $[24,25]$. Although the insomnia-low physical activity association has been well studied, the relationship between insomnia and CRF remains less explored. Regular moderate to vigorous exercise may increase sleep time and improve sleep consolidation through mechanisms such as altered body core temperature and increased brain-derived neurotropic factor activity [26]. Physical exercise induces an acute rise, but over longer term reduction, of growth hormone and testosterone levels. The magnitude of this response is influenced by factors such as previous exercise levels, age, and nutritional status [27, 28]. The role of an increase in growth hormone level for the subsequent night sleep is inconclusive and it remains uncertain whether physical exercise can alter the circadian fluctuations in hormone levels including testosterone and cortisol [29]. Nevertheless, elevated sympathetic activity, increased cortisol levels, and inflammation caused by insomnia may lead to lowered alertness, mood disorders, and higher rate of perceived exertion which may limit physical performance by reduced effort [26].

Indeed, a lower CRF in insomnia, as compared with noninsomnia subjects, was observed in the current study indicating that insomnia as a chronic disease may influence the complex biological processes involving the integrative coordination of multiple physiological systems for $\mathrm{O}_{2}$ metabolism and transport. In gender-specific analyses, an inverse association between insomnia and CRF was found in men but not in women. The exact explanation of this gender-specific effect is unclear. However, it is known that subjects who participated in the fitness test are generally healthier than those not participating, due to the exclusion criteria applied for the exercise test. In the current study, the prevalence of insomnia was lower in the included women compared to women excluded from the analyses. This may have reduced the power to detect a significant association between insomnia and CRF in women.

The ISI used in the current study provides a more comprehensive assessment of nighttime and daytime components of insomnia compared with information on insomnia symptoms alone. An ISI cutoff of 10 has been shown to detect insomnia cases in a community sample with $86 \%$ sensitivity and $88 \%$ specificity [16]. The prevalence of insomnia in the current study is comparable to that reported in a Swedish population-based survey [30]. In line with other epidemiological studies, we found that female gender, low social economy status, no income-related job, and depression symptoms were overrepresented in the insomnia group [1]. In our study, more than $20 \%$ of the subjects with insomnia reported a sleep duration of less than $6 \mathrm{~h}$ similar to another population-based study [31]. Short sleep duration has been shown to independently predict cardiovascular morbidity $[5,32]$, and it was argued that studies on insomnia and cardiovascular disease should take into account the impact of short habitual sleep time [33]. Therefore, we included subjective short sleep duration in our multivariate analysis and an independent association between insomnia and CRF remained, in men.

The observed difference $\left(1.15 \mathrm{ml}^{*} \mathrm{~kg}^{-1} * \mathrm{~min}^{-1}\right)$ in CRF between the male insomnia and non-insomnia groups is modest but of clinical relevance. The age-related decline in $\mathrm{VO}_{2} \max$ has been reported to be $0.26 \mathrm{ml}^{*} \mathrm{~kg}^{-1} * \min ^{-} 1$ per year in men 
Table 2 Multivariate generalized linear model for $\mathrm{VO}_{2}$ max prediction in the full sample $(n=603)$

\begin{tabular}{lcr}
\hline & $\beta$-coefficients $(95 \% \mathrm{CI})$ & $p$ value \\
\hline Men vs. women & $7.47(6.64-8.30)$ & $<0.001$ \\
Age $($ years $)$ & $-0.32(-0.40--0.25)$ & $<0.001$ \\
BMI $\left(\mathrm{kg} / \mathrm{m}^{2}\right)$ & $-0.50(-0.66-0.33)$ & $<0.001$ \\
Waist circumference (cm) & $-0.16(-0.22--0.10)$ & $<0.001$ \\
Low vs. high socioeconomic status & $-1.30(-2.02--0.59)$ & $<0.001$ \\
With vs. without university education & $0.97(0.27-1.67)$ & 0.007 \\
Without vs. with income-related job & $0.68(-0.24-1.60)$ & 0.146 \\
Smoking & & 0.257 \\
Occasional vs. never & $1.15(-0.84-3.13)$ & 0.753 \\
Former vs. never & $-0.12(-0.83-0.60)$ & 0.978 \\
Current vs. never & $0.02(-1.14-1.18)$ & 0.265 \\
Risky vs. not risky alcohol consumption & $0.42(-0.32-1.16)$ & 0.148 \\
Insomnia vs. non-insomnia & $-0.56(-1.31-0.20)$ & 0.377 \\
Subjective sleep duration $<6$ h vs. $\geq 6$ h & $0.55(-0.66-1.75)$ & 0.071 \\
Sleep apnea vs. no apnea & $1.51(-0.13-3.15)$ & 0.390 \\
Chronic disease vs. no disease & $-0.45(-1.49-0.58)$ & 0.293 \\
Depression symptoms vs. no symptoms & $0.43(-0.37-1.23)$ & $<0.001$ \\
Percentage (\%) MVPA & $0.24(0.12-0.37)$ & 0.541 \\
Percentage (\%) SED & $0.01(-0.03-0.05)$ & \\
\hline
\end{tabular}

Chronic disease includes stroke, coronary artery disease, diabetes, cancer, and COPD

Significant $p$ value presented in italics

$M V P A$ moderate to vigorous intensity physical activity, $S E D$ time spent sedentary
[34]. A meta-analysis of 102,980 healthy men and women indicated that each $3.5 \mathrm{ml} * \mathrm{~kg}^{-1} * \mathrm{~min}^{-1}$ increase in CRF is associated with a $13 \%$ lower risk of all-cause mortality and $15 \%$ lower risk of cardiovascular morbidity [11]. It has also been shown that men with CRF corresponding to the lowest quintile have a substantially greater risk for all-cause mortality as compared with men with moderate or high levels of CRF $[35,36]$. A recent statement from the American Heart Association has emphasized the importance of CRF assessment in clinical practice, either by measurement or estimation, in order to optimize the prevention and treatment of cardiovascular disease [10]. In the current study, a submaximal cycle ergometer test was applied instead of a maximal protocol with ventilatory gas analyses in order to increase the feasibility of the protocol in the population-based setting. A newly developed equation with improved validity was used for $\mathrm{VO}_{2} \max$ estimation [19]. It is believed that this approach enables a more representative cohort and increases the generalizability of the result.

Despite the gender-balanced design, inclusion of submaximal exercise testing and objectively measured physical activity, our study also comes with limitations. Despite the fact that ISI is a valid diagnostic instrument for insomnia, the intensity and duration of the disease are not addressed. Although adequate, the use of an ISI cutoff of 10 could have led to an identification of more subjects with mild insomnia in the current study. This could, in part, have attributed to the small difference in $\mathrm{VO}_{2} \mathrm{max}$ observed in the insomnia and non-insomnia groups. Depression symptoms and sleep apnea were assessed by single questions rather than by validated questionnaires. The cross-sectional design of the current study does not allow any conclusions on causality for the relationship between insomnia and CRF. It has recently been shown that a decline in CRF increases the odds of incident sleep complaints [37]. Whether the observed difference in $\mathrm{VO}_{2}$ max between insomnia and non-insomnia men is due to altered physiological adaptation to physical activity needs to be further studied. Finally, the age range of the cohort may limit the generalizability of the results. Prospective studies with larger age range and detailed mapping of insomnia

Table 3 Multivariate generalized linear model for VO2max prediction stratified by gender

\begin{tabular}{lll}
\hline & Men $(n=289)$ & \\
& $\beta$-coefficient $(95 \% \mathrm{CI})$ & $p$ value \\
Insomnia vs. non-insomnia & $-1.15(-2.23--0.06)$ & 0.038 \\
& Women $(n=314)$ & \\
& $\beta$-coefficient $(95 \% \mathrm{CI})$ & $p$ value \\
Insomnia vs. non-insomnia & $-0.09(-1.09-0.92)$ & 0.866 \\
\hline
\end{tabular}

Adjusting for age, body mass index, waist circumference, socioeconomic status, university education, income-related job, smoking, risky drinking, chronic disease (including stroke, coronary artery disease, diabetes, cancer, and COPD), sleep apnea, depression symptoms, sleep duration $<6 \mathrm{~h}$, percentage of moderate to vigorous intensity physical activity, and percentage of time spent sedentary

Significant $p$ value presented in italics 
subtype are warranted to further elucidate the relationship between insomnia and CRF.

\section{Conclusion}

In this urban mid-aged Swedish population, we demonstrated that insomnia is associated with lower CRF in men, independent of body composition, living conditions, comorbidity, and lifestyle, including objectively assessed physical activity. CRF represents the body's adaptability to physical exertion which may be limited by insomnia. In light of the strong association between low CRF and increased cardiometabolic disease risk, our results provide a possible link of insomnia to cardiovascular morbidity and mortality.

Acknowledgements The authors would like to acknowledge AnnChristin Lundqvist RN and Lena Engelmark RN for data collection and data entry and Dr. Mio Frisk for valuable comments on the manuscript. The main funding body of The Swedish CArdioPulmonary bioImage Study (SCAPIS) is the Swedish Heart and Lung Foundation. The study is also funded by the Knut and Alice Wallenberg Foundation, the Swedish Research Council and VINNOVA (Sweden's Innovation agency). In addition, the SCAPIS Pilot study received support from the Sahlgrenska Academy at University of Gothenburg and Region Västra Götaland. We are very grateful to all the participants in this study and the staff at the SCAPIS test center in Gothenburg.

Funding information The SCAPIS study was funded by Swedish Heart and Lung Foundation, Knut and Alice Wallenberg Foundation, the Swedish Research Council and VINNOVA (Sweden's Innovation agency). In addition, the SCAPIS Pilot study received support from the Sahlgrenska Academy at University of Gothenburg and Region Västra Götaland.

\section{Compliance with ethical standards}

Conflict of interest JH reports grants from Swedish Heart and Lung Foundation (grant number 20120429), and from the University of Göteborg during the conduct of the study. DZ, HW, ÖE, LG, DA, AB, $\mathrm{KT}, \mathrm{GB}$, and MB declare no conflict of interest related to this work.

Ethical approval All procedures performed in studies involving human participants were in accordance with the ethical standards of the institutional and/or national research committee and with the 1964 Helsinki declaration and its later amendments or comparable ethical standards.

Informed consent Informed consent was obtained from all individual participants included in the study.

Open Access This article is distributed under the terms of the Creative Commons Attribution 4.0 International License (http:// creativecommons.org/licenses/by/4.0/), which permits unrestricted use, distribution, and reproduction in any medium, provided you give appropriate credit to the original author(s) and the source, provide a link to the Creative Commons license, and indicate if changes were made.

Publisher's note Springer Nature remains neutral with regard to jurisdictional claims in published maps and institutional affiliations.

\section{References}

1. Ohayon MM (2002) Epidemiology of insomnia: what we know and what we still need to learn. Sleep Med Rev 6:97-111

2. Ford ES, Cunningham TJ, Giles WH, Croft JB (2015) Trends in insomnia and excessive daytime sleepiness among U.S. adults from 2002 to 2012. Sleep Med 16:372-378

3. Pallesen S, Sivertsen B, Nordhus IH, Bjorvatn B (2014) A 10-year trend of insomnia prevalence in the adult Norwegian population. Sleep Med 15:173-179

4. Kronholm E, Partonen T, Härmä M, Hublin C, Lallukka T, Peltonen M, Laatikainen T (2016) Prevalence of insomnia-related symptoms continues to increase in the Finnish working-age population. J Sleep Res 25:454-457

5. St-Onge MP, Grandner MA, Brown D, Conroy MB, Jean-Louis G, Coons M, Bhatt DL, American Heart Association Obesity, Behavior Change, Diabetes, and Nutrition Committees of the Council on Lifestyle and Cardiometabolic Health; Council on Cardiovascular Disease in the Young; Council on Clinical Cardiology; and Stroke Council (2016) Sleep duration and quality: impact on lifestyle behaviors and cardiometabolic health: a scientific statement from the American Heart Association. Circulation 134:e367-e386

6. Javaheri S, Redline S (2017) Insomnia and risk of cardiovascular disease. Chest 152:435-444

7. Routledge FS, Dunbar SB, Higgins M, Rogers AE, Feeley C, Ioachimescu O, Euwer K, Eapen D, Quyyumi A (2017) Insomnia symptoms are associated with abnormal endothelial function. J Cardiovasc Nurs 32:78-85

8. Haario P et al (2013) Bidirectional associations between insomnia symptoms and unhealthy behaviours. J Sleep Res 22:89-95

9. Caspersen CJ, Powell KE, Christenson GM (1985) Physical activity, exercise, and physical fitness: definitions and distinctions for health-related research. Public Health Rep 100:126-131

10. Ross R, Blair SN, Arena R, Church TS, Després JP, Franklin BA, Haskell WL, Kaminsky LA, Levine BD, Lavie CJ, Myers J, Niebauer J, Sallis R, Sawada SS, Sui X, Wisløff U, American Heart Association Physical Activity Committee of the Council on Lifestyle and Cardiometabolic Health, Council on Clinical Cardiology, Council on Epidemiology and Prevention, Council on Cardiovascular and Stroke Nursing, Council on Functional Genomics and Translational Biology, Stroke Council (2016) Importance of assessing cardiorespiratory fitness in clinical practice: a case for fitness as a clinical vital sign: a scientific statement from the American Heart Association. Circulation 134:e653-e699

11. Kodama S, Saito K, Tanaka S, Maki M, Yachi Y, Asumi M, Sugawara A, Totsuka K, Shimano H, Ohashi Y, Yamada N, Sone H (2009) Cardiorespiratory fitness as a quantitative predictor of allcause mortality and cardiovascular events in healthy men and women: a meta-analysis. JAMA 301:2024-2035

12. Strand LB, Laugsand LE, Wisløff U, Nes BM, Vatten L, Janszky I (2013) Insomnia symptoms and cardiorespiratory fitness in healthy individuals: the Nord-Trondelag Health Study (HUNT). Sleep 36: 99-108

13. Gerber M et al (2010) Fitness and exercise as correlates of sleep complaints: is it all in our minds? Med Sci Sports Exerc 42:893901

14. Bergstrom G et al (2015) The Swedish CArdioPulmonary BioImage study: objectives and design. J Intern Med 278:645-659

15. Bastien CH, Vallieres A, Morin CM (2001) Validation of the Insomnia Severity Index as an outcome measure for insomnia research. Sleep Med 2:297-307

16. Morin CM, Belleville G, Bélanger L, Ivers H (2011) The insomnia severity index: psychometric indicators to detect insomnia cases and evaluate treatment response. Sleep 34:601-608 
17. Ekblom-Bak E, Björkman F, Hellenius ML, Ekblom B (2014) A new submaximal cycle ergometer test for prediction of VO2max. Scand J Med Sci Sports 24:319-326

18. Dunbar CC, Robertson RJ, Baun R, Blandin MF, Metz K, Burdett R, Goss FL (1992) The validity of regulating exercise intensity by ratings of perceived exertion. Med Sci Sports Exerc 24:94-99

19. Bjorkman F et al (2016) Validity of the revised Ekblom Bak cycle ergometer test in adults. Eur J Appl Physiol 116:1627-1638

20. Ekblom-Bak E, Olsson G, Ekblom Ö, Ekblom B, Bergström G, Börjesson M (2015) The daily movement pattern and fulfilment of physical activity recommendations in Swedish Middle-Aged Adults: the SCAPIS Pilot Study. PLoS One 10:e0126336

21. Aguilar-Farias N, Brown WJ, Peeters GM (2014) ActiGraph GT3X+ cut-points for identifying sedentary behaviour in older adults in free-living environments. J Sci Med Sport 17:293-299

22. Sasaki JE, John D, Freedson PS (2011) Validation and comparison of ActiGraph activity monitors. J Sci Med Sport 14:411-416

23. Lundin A, Hallgren M, Balliu N, Forsell Y (2015) The use of alcohol use disorders identification test (AUDIT) in detecting alcohol use disorder and risk drinking in the general population: validation of AUDIT using schedules for clinical assessment in neuropsychiatry. Alcohol Clin Exp Res 39:158-165

24. Williams PT (2001) Physical fitness and activity as separate heart disease risk factors: a meta-analysis. Med Sci Sports Exerc 33:754761

25. DeFina LF, Haskell WL, Willis BL, Barlow CE, Finley CE, Levine BD, Cooper KH (2015) Physical activity versus cardiorespiratory fitness: two (partly) distinct components of cardiovascular health? Prog Cardiovasc Dis 57:324-329

26. Chennaoui M, Arnal PJ, Sauvet F, Léger D (2015) Sleep and exercise: a reciprocal issue? Sleep Med Rev 20:59-72

27. Kraemer WJ, Dunn-Lewis C, Comstock BA, Thomas GA, Clark JE, Nindl BC (2010) Growth hormone, exercise, and athletic performance: a continued evolution of complexity. Curr Sports Med Rep 9:242-252

28. Hackney AC (1996) The male reproductive system and endurance exercise. Med Sci Sports Exerc 28:180-189

29. Teo W, Newton MJ, McGuigan MR (2011) Circadian rhythms in exercise performance: implications for hormonal and muscular adaptation. J Sports Sci Med 10:600-606
30. Ohayon MM, Bader G (2010) Prevalence and correlates of insomnia in the Swedish population aged 19-75 years. Sleep Med 11:980986

31. Ohayon MM, Sagales T (2010) Prevalence of insomnia and sleep characteristics in the general population of Spain. Sleep Med 11: 1010-1018

32. Cappuccio FP, Cooper D, D'Elia L, Strazzullo P, Miller MA (2011) Sleep duration predicts cardiovascular outcomes: a systematic review and meta-analysis of prospective studies. Eur Heart J 32: 1484-1492

33. Grandner MA, Perlis ML (2013) Insomnia as a cardiometabolic risk factor. Sleep 36:11-12

34. Eriksen L, Grønbaek M, Helge JW, Tolstrup JS (2016) Cardiorespiratory fitness in 16025 adults aged 18-91 years and associations with physical activity and sitting time. Scand J Med Sci Sports 26:1435-1443

35. Farrell SW et al (2015) Is there a gradient of mortality risk among men with low cardiorespiratory fitness? Med Sci Sports Exerc 47: 1825-1832

36. Blair SN, Kohl HW 3rd, Paffenbarger RS Jr, Clark DG, Cooper KH, Gibbons LW (1989) Physical fitness and all-cause mortality. A prospective study of healthy men and women. JAMA 262:23952401

37. Dishman RK et al (2015) Decline in cardiorespiratory fitness and odds of incident sleep complaints. Med Sci Sports Exerc 47:960 966

\section{Comments}

This study shows in a relatively large cohort of the middle-aged population (50-64 years) the small but for public health relevant effect of lower cardiorespiratory fitness in men with insomina independent of physical activity sedentary time. The reason of non-association in women remains unclear and should be a focus in further research.

Bernd Wolfarth

Berlin, Germany 